\title{
Strong Convergence Theorems for Variational Inequalities and Split Equality Problem
}

\author{
Yu Jing Wu, ${ }^{1}$ Ru Dong Chen, ${ }^{2}$ and Luo Yi Shi ${ }^{2}$ \\ ${ }^{1}$ Tianjin Vocational Institute, Tianjin 300410, China \\ ${ }^{2}$ Department of Mathematics, Tianjin Polytechnic University, Tianjin 300387, China
}

Correspondence should be addressed to Ru Dong Chen; chenrd@tjpu.edu.cn

Received 5 July 2013; Revised 22 August 2013; Accepted 2 September 2013

Academic Editor: Dashan Fan

Copyright (C) $2013 \mathrm{Yu}$ Jing Wu et al. This is an open access article distributed under the Creative Commons Attribution License, which permits unrestricted use, distribution, and reproduction in any medium, provided the original work is properly cited.

Let $H_{1}, H_{2}$, and $H_{3}$ be real Hilbert spaces, let $C \subseteq H_{1}, Q \subseteq H_{2}$ be two nonempty closed convex sets, and let $A: H_{1} \rightarrow H_{3}, B: H_{2} \rightarrow$ $H_{3}$ be two bounded linear operators. The split equality problem (SEP) is to find $x \in C, y \in Q$ such that $A x=B y$. Let $H=H_{1} \times H_{2}$; consider $f: H \rightarrow H$ a contraction with coefficient $0<\alpha<1$, a strongly positive linear bounded operator $T: H \rightarrow H$ with coefficient $\bar{\gamma}>0$, and $M: H \rightarrow H$ is a $\beta$-inverse strongly monotone mapping. Let $0<\gamma<\bar{\gamma} / \alpha, S=C \times Q$ and $G: H \rightarrow H_{3}$ be defined by restricting to $H_{1}$ is $A$ and restricting to $H_{2}$ is $-B$, that is, $G$ has the matrix form $G=[A,-B]$. It is proved that the sequence $\left\{w_{n}\right\}=\left\{\left(x_{n}, y_{n}\right)\right\} \subseteq H$ generated by the iterative method $w_{n+1}=P_{S}\left[\alpha_{n} \gamma f\left(w_{n}\right)+\left(I-\alpha_{n} T\right) P_{S}\left(I-\gamma_{n} G^{*} G\right) P_{S}\left(w_{n}-\lambda_{n} M w_{n}\right)\right]$ converges strongly to $\widetilde{w}$ which solves the SEP and the following variational inequality: $\langle(T-\lambda f) \widetilde{w}, w-\widetilde{w}\rangle \geq 0$ and $\langle M \widetilde{w}, w-\widetilde{w}\rangle \geq 0$ for all $w \in S$. Moreover, if we take $M=G^{*} G: H \rightarrow H, \gamma_{n}=0$, then $M$ is a $\beta$-inverse strongly monotone mapping, and the sequence $\left\{w_{n}\right\}$ generated by the iterative method $w_{n+1}=\alpha_{n} \gamma f\left(w_{n}\right)+\left(I-\alpha_{n} T\right) P_{S}\left(w_{n}-\lambda_{n} G^{*} G w_{n}\right)$ converges strongly to $\widetilde{w}$ which solves the SEP and the following variational inequality: $\langle(T-\lambda f) \widetilde{w}, w-\widetilde{w}\rangle \geq 0$ for all $w \in S$.

\section{Introduction and Preliminaries}

Throughout this paper, let $H$ be a real Hilbert space and $S$ a nonempty closed convex subset of $H$, and let $I$ denote the identity operator on Hilbert space $H, \operatorname{Fix}(T)$ the set of the fixed points of an operator $T$. An operator $T$ on a Hilbert space $H$ is nonexpansive if, for each $x$ and $y$ in $H,\|T x-T y\| \leq$ $\|x-y\| . T$ is said to be strongly positive, if there exists $\bar{\gamma}>0$ such that $\langle T x, x\rangle \geq \bar{\gamma}\|x\|^{2}$ for all $x \in H$. T is said to be inverse strongly monotone if there exists $\beta>0$ such that $\langle x-y, T x-T y\rangle \geq \beta\|T x-T y\|^{2}$ for all $x, y \in H$. A bounded linear operator $T$ on a Hilbert space $H$ is positive operator if $T^{*}=T$ and $\lambda \geq 0$ for all $\lambda$ in the spectrum of $T$ or equivalent to $\langle T x, x\rangle \geq 0$ for all $x \in H$.

Let $P_{S}$ denote the projection from $H$ onto a nonempty closed convex subset $S$ of $H$; that is,

$$
P_{S}(w)=\min _{x \in S}\|x-w\|
$$

It is well known that $P_{S}(w)$ is characterized by the inequality

$$
\left\langle w-P_{S}(w), x-P_{S}(w)\right\rangle \leq 0, \quad \forall x \in S,
$$

and $P_{S}$ is nonexpansive.

Let $C$ and $Q$ be nonempty closed convex subsets of real Hilbert spaces $H_{1}$ and $H_{2}$, respectively, and let $A: H_{1} \rightarrow$ $\mathrm{H}_{2}$ be a bounded linear operator. The split feasibility problem (SFP) is to find a point $x$ satisfying the property

$$
x \in C, \quad A x \in Q \text {. }
$$

The SFP was first introduced by Censor and Elfving [1], which attracts many authors' attention due to its application in signal processing [1]. Various algorithms have been invented to solve it (see [2-7]).

Recently, Moudafi [8] proposes a new split equality problem (SEP): let $H_{1}, H_{2}$, and $H_{3}$ be real Hilbert spaces, let $C \subseteq H_{1}, Q \subseteq H_{2}$ be two nonempty closed convex sets, and 
let $A: H_{1} \rightarrow H_{3}, B: H_{2} \rightarrow H_{3}$ be two bounded linear operators. Find $x \in C, y \in Q$ satisfying

$$
A x=B y .
$$

When $B=I$, SEP reduces to the well-known SFP. In the paper [8], Moudafi gives the alternating CQ-algorithm and relaxed alternating CQ-algorithm iterative algorithm to solve the split equality problem. We must point out that the above algorithms converge weakly to a solution of the SEP.

We use $\Gamma=\left\{(x, y) \in H_{1} \times H_{2}, A x=B y, x \in C, y \in Q\right\}$ to denote the solution set of SEP, and letting $S=C \times Q$ in $H=H_{1} \times H_{2}$ define $G: H \rightarrow H_{3}$ by $G=[A,-B]$, then $G^{*} G: H \rightarrow H$ has the matrix form

$$
G^{*} G=\left[\begin{array}{cc}
A^{*} A & -A^{*} B \\
-B^{*} A & B^{*} B
\end{array}\right] .
$$

The original problem can now be reformulated as finding $w=(x, y) \in S$ with $G w=0$ or, more generally, minimizing the function $\|G w\|$ over $w \in S$. Therefore solving SEP (4) is equivalent to solving the following minimization problem:

$$
\min _{w \in S} f(w)=\frac{1}{2}\|G w\|^{2}
$$

In the paper [9], we use the well-known Tychonov regularization to get some algorithms converge strongly to the minimum-norm solution of the SEP. Moreover, in the paper [9], we obtain that, for $0<\gamma<2 /\|G\|^{2}, \operatorname{Fix}\left(P_{S}\left(I-\gamma G^{*} G\right)\right)=$ $\operatorname{Fix}\left(P_{S}\right) \cap \operatorname{Fix}\left(I-\gamma G^{*} G\right)=\Gamma$. That is to say, the set of the solution of SEP is equal to the fixed points set of the nonexpansive mapping $P_{S}\left(I-\gamma G^{*} G\right)$.

Recall that the variational inequality problem is to find $p \in H$ such that $\langle M p, x-p\rangle \geq 0$, for all $x \in S$, where $S$ is closed, convex, and nonempty, and $M$ is a mapping on $H$. The set of solutions of the variational inequality is denoted by $\mathrm{VI}(S, M)$. In the paper [9], we also get that $w \in \operatorname{Fix}\left(P_{S}(I-\right.$ $\left.\gamma G^{*} G\right)$ ) if and only if $w$ is a solution of the variational inequality $\left\langle G^{*} G w, v-w\right\rangle \geq 0$, for all $v \in S$.

In this paper, we consider a contraction $f$ on $H=H_{1} \times$ $\mathrm{H}_{2}$ with coefficient $0<\alpha<1$, a strongly positive linear bounded operator $T$ with coefficient $\bar{\gamma}>0$, and $M$ is a $\beta$ inverse strongly monotone mapping. Let $0<\gamma<\bar{\gamma} / \alpha$, $S=C \times Q$. We prove that the sequence $\left\{w_{n}\right\}=\left\{\left(x_{n}, y_{n}\right)\right\} \subseteq H$ generated by the iterative method $w_{n+1}=P_{S}\left[\alpha_{n} \gamma f\left(w_{n}\right)+(I-\right.$ $\left.\left.\alpha_{n} T\right) P_{S}\left(I-\gamma_{n} G^{*} G\right) P_{S}\left(w_{n}-\lambda_{n} M w_{n}\right)\right]$ converges strongly to $\widetilde{w}$ which solves the SEP and the following variational inequality: $\langle(A-\lambda f) \widetilde{w}, w-\widetilde{w}\rangle \geq 0$ and $\langle M \widetilde{w}, w-\widetilde{w}\rangle \geq 0$ for all $w \in S$ under some mild conditions for $\alpha_{n}, \gamma_{n}$, and $\lambda_{n}$.

We now collect some elementary facts which will be used in the proofs of our main results.

Lemma 1. Assuming $T$ is a positive operator, then $T$ is $1 /\|T\|-$ inverse strongly monotone.

Proof. Since $T$ is a positive operator on $H$, by the functional calculus of positive operator (for more details see [10, Chapter $4]$ ), there exists positive operator $T_{1}$ such that $T=T_{1} T_{1}$ and $\left\|T_{1}\right\|=\|T\|^{1 / 2}$.
Hence, for any $x, y \in H,\|T x-T y\|^{2}=\left\|T_{1} T_{1}(x-y)\right\|^{2} \leq$ $\left\|T_{1}\right\|^{2}\left\|T_{1}(x-y)\right\|^{2}=\|T\|\left\langle T_{1}(x-y), T_{1}(x-y)\right\rangle=\|T\|\langle x-$ $\left.y, T_{1} T_{1}(x-y)\right\rangle=\|T\|\langle x-y, T x-T y\rangle$. It follows that $\langle x-$ $y, T x-T y\rangle \geq(1 /\|T\|)\|T x-T y\|^{2}$. That is to say, $T$ is $1 /\|T\|-$ inverse strongly monotone.

Lemma 2 (see $[11,12])$. Let $X$ be a Banach space, $C$ a closed convex subset of $X$, and $T: C \rightarrow C$ a nonexpansive mapping with $\operatorname{Fix}(T) \neq \emptyset$. If $\left\{x_{n}\right\}$ is a sequence in $C$ weakly converging to $x$ and if $\left\{(I-T) x_{n}\right\}$ converges strongly to $y$, then $(I-T) x=y$.

Lemma 3 (see [13]). Let $\left\{s_{n}\right\}$ be a sequence of nonnegative real numbers, $\left\{\alpha_{n}\right\}$ a sequence of real numbers in $[0,1]$ with $\sum_{n=1}^{\infty} \alpha_{n}=\infty,\left\{u_{n}\right\}$ a sequence of nonnegative real numbers with $\sum_{n=1}^{\infty} u_{n}<\infty$, and $\left\{t_{n}\right\}$ a sequence of real numbers with $\lim \sup _{n} t_{n} \leq 0$. Suppose that

$$
s_{n+1}=\left(1-\alpha_{n}\right) s_{n}+\alpha_{n} t_{n}+u_{n}, \quad \forall n \in \mathbb{N}
$$

Then $\lim _{n \rightarrow \infty} s_{n}=0$.

Lemma 4 (see [14]). Assuming $T$ is a strongly positive linear bounded operator on a Hilbert space $H$ with coefficient $\bar{\gamma}>0$, then $\|I-\rho T\| \leq 1-\rho \bar{\gamma}$ for $0<\rho \leq\|T\|^{-1}$.

Lemma 5 (see [13]). Let $S$ be a nonempty and closed subset of a Banach space $X$, and let $\left\{R_{n}\right\}$ be a family of mappings of $S$ into itself which satisfies the condition: for each bounded subset E of $S$,

$$
\sum_{n=1}^{\infty} \sup \left\{\left\|R_{n+1} w-R_{n} w\right\|, w \in E\right\}<\infty .
$$

Then, for each $w \in S,\left\{R_{n} w\right\}$ converges strongly to a point in $S$. Moreover, $R$ is defined by

$$
R w=\lim _{n \rightarrow \infty} R_{n} w, \quad w \in E .
$$

Then, for each bounded subset $E$ of $S$,

$$
\limsup _{n \rightarrow \infty}\left\{\left\|R w-R_{n} w\right\|, w \in E\right\}=0
$$

and $\operatorname{Fix}(R)=\cap \operatorname{Fix}\left(R_{n}\right)$, if $\cap \operatorname{Fix}\left(R_{n}\right) \neq \emptyset$.

\section{Main Results}

Theorem 6. Let $\Gamma$ denote the solutions set of the $S E P, S=C \times Q$, $f$ a contraction on $H=H_{1} \times H_{2}$ with coefficient $0<\alpha<1$, a strongly positive linear bounded operator $T$ with coefficient $\bar{\gamma}>0$, and $M$ a $\beta$-inverse strongly monotone mapping. Assume that $0<\gamma<\bar{\gamma} / \alpha, \Gamma \cap V I(S, M) \neq \emptyset$, and $\left\{w_{n}\right\}=\left\{\left(x_{n}, y_{n}\right)\right\} \subseteq H$ is the sequence generated by the following algorithm: $w_{0} \in H$,

$$
\begin{aligned}
w_{n+1}=P_{S}[ & \alpha_{n} \gamma f\left(w_{n}\right) \\
& \left.+\left(I-\alpha_{n} T\right) P_{S}\left(I-\gamma_{n} G^{*} G\right) P_{S}\left(w_{n}-\lambda_{n} M w_{n}\right)\right]
\end{aligned}
$$


for all $n=0,1,2, \ldots$, where $\alpha_{n} \in(0,1), \lambda_{n} \in(0,2 \beta)$, and $\gamma_{n} \in$ $\left(0,2 /\|G\|^{2}\right)$. If $\alpha_{n}, \lambda_{n}$, and $\gamma_{n}$ are chosen such that $\lambda_{n} \in[a, b]$ for some $a, b$ with $0<a<b<2 \beta$ and

(i) $\lim _{n} \alpha_{n}=0$,

(ii) $\sum_{n=0}^{\infty} \alpha_{n}=\infty$,

(iii) $\sum_{n=0}^{\infty}\left|\alpha_{n+1}-\alpha_{n}\right|<\infty$,

(v) $\sum_{n=0}^{\infty}\left|\lambda_{n+1}-\lambda_{n}\right|<\infty$,

(iv) $\sum_{n=0}^{\infty}\left|\gamma_{n+1}-\gamma_{n}\right|<\infty$,

then the sequence $\left\{w_{n}\right\}$ converges strongly to $\widetilde{w}$ which solves the SEP and the following variational inequality: $\langle(T-\lambda f) \widetilde{w}, w-$ $\widetilde{w}\rangle \geq 0$ and $\langle M \widetilde{w}, w-\widetilde{w}\rangle \geq 0$ for all $w \in S$.

Proof. First, we show that the sequence $\left\{w_{n}\right\}$ is bounded. Consider the mapping $I-\lambda_{n} M$. Since $M$ is a $\beta$-inverse strongly monotone mapping, we have that, for all $w, v \in S$,

$$
\begin{aligned}
\|(I- & \left.\lambda_{n} M\right) w-\left(I-\lambda_{n} M\right) v \|^{2} \\
= & \left\|(w-v)-\lambda_{n}(M w-M v)\right\|^{2} \\
= & \|w-v\|^{2}-2 \lambda_{n}\langle w-v, M w-M v\rangle \\
& +\lambda_{n}^{2}\|M w-M v\|^{2} \\
\leq & \|w-v\|^{2}+\lambda_{n}\left(\lambda_{n}-2 \beta\right)\|M w-M v\|^{2} .
\end{aligned}
$$

Since $0<\lambda_{n}<2 \beta$, we deduce that $\|\left(I-\lambda_{n} M\right) w-(I-$ $\left.\lambda_{n} M\right) v\left\|^{2} \leq\right\| w-v \|^{2}$. That is to say, $I-\lambda_{n} M$ is nonexpansive.

Letting $v_{n}=P_{S}\left(w_{n}-\lambda_{n} M w_{n}\right), R_{n}=P_{S}\left(I-\gamma_{n} G^{*} G\right)$, choosing $u \in \Gamma \cap \operatorname{VI}(S, M)$, then $u=P_{S}\left(I-\gamma G^{*} G\right) u$ and $u=P_{S}\left(I-\lambda_{n} M\right) \mathcal{u} . P_{S}$ is nonexpansive, which implies that

$$
\begin{aligned}
\left\|v_{n}-u\right\| & =\left\|P_{S}\left(w_{n}-\lambda_{n} M w_{n}\right)-P_{S}\left(u-\lambda_{n} M u\right)\right\| \\
& \leq\left\|\left(w_{n}-\lambda_{n} M w_{n}\right)-\left(u-\lambda_{n} M u\right)\right\| \\
& =\left\|\left(I-\lambda_{n} M\right) w_{n}-\left(I-\lambda_{n} M\right) u\right\| \\
& \leq\left\|w_{n}-u\right\|, \\
\left\|w_{n+1}-u\right\| & =\left\|P_{S}\left[\alpha_{n} \gamma f\left(w_{n}\right)+\left(I-\alpha_{n} T\right) R_{n} v_{n}\right]-u\right\| \\
& \leq\left\|\alpha_{n} \gamma f\left(w_{n}\right)+\left(I-\alpha_{n} T\right) R_{n} v_{n}-u\right\| \\
& =\left\|\alpha_{n}\left(\gamma f\left(w_{n}\right)-T u\right)+\left(I-\alpha_{n} T\right)\left(R_{n} v_{n}-u\right)\right\| .
\end{aligned}
$$

Since $T$ is strongly positive linear bounded operator, $R_{n}$ is nonexpansive, and $f$ is contraction, we have

$$
\begin{aligned}
\left\|w_{n+1}-u\right\| \leq & \alpha_{n}\left\|\gamma f\left(w_{n}\right)-T u\right\|+\left(1-\alpha_{n} \bar{\gamma}\right)\left\|R_{n} v_{n}-u\right\| \\
\leq & \alpha_{n}\left\|\gamma f\left(w_{n}\right)-\gamma f(u)\right\|+\alpha_{n}\|\gamma f(u)-T u\| \\
& +\left(1-\alpha_{n} \bar{\gamma}\right)\left\|R_{n} v_{n}-u\right\| \\
\leq & \alpha \gamma \alpha_{n}\left\|w_{n}-u\right\|+\alpha_{n}\|\gamma f(u)-T u\| \\
& +\left(1-\alpha_{n} \bar{\gamma}\right)\left\|R_{n} v_{n}-u\right\|
\end{aligned}
$$

$$
\begin{aligned}
= & \alpha \gamma \alpha_{n}\left\|w_{n}-u\right\|+\alpha_{n}\|\gamma f(u)-T u\| \\
& +\left(1-\alpha_{n} \bar{\gamma}\right)\left\|R_{n} v_{n}-R_{n} u\right\| \\
\leq & \alpha \gamma \alpha_{n}\left\|w_{n}-u\right\|+\alpha_{n}\|\gamma f(u)-T u\| \\
& +\left(1-\alpha_{n} \bar{\gamma}\right)\left\|v_{n}-u\right\| \\
\leq & \alpha \gamma \alpha_{n}\left\|w_{n}-u\right\|+\alpha_{n}\|\gamma f(u)-T u\| \\
& +\left(1-\alpha_{n} \bar{\gamma}\right)\left\|w_{n}-u\right\| \\
= & \left(1-\alpha\left(\bar{\gamma}-\gamma \alpha_{n}\right)\right)\left\|w_{n}-u\right\| \\
& +\alpha_{n}\|\gamma f(u)-T u\| \\
\leq & \max \left\{\left\|w_{n}-u\right\|, \frac{1}{\bar{\gamma}-\gamma \alpha_{n}}\|\gamma f(u)-T u\|\right\} .
\end{aligned}
$$

It follows from induction that

$$
\begin{array}{r}
\left\|w_{n}-u\right\| \leq \max \left\{\left\|w_{0}-u\right\|, \frac{1}{\bar{\gamma}-\gamma \alpha_{n}}\|\gamma f(u)-T u\|\right\}, \\
n>0 .
\end{array}
$$

Hence, $\left\{w_{n}\right\}$ is bounded, so are $\left\{v_{n}\right\},\left\{R_{n} v_{n}\right\},\left\{M w_{n}\right\}$, and $\left\{f\left(w_{n}\right)\right\}$.

Next, we show that $\left\|w_{n+1}-w_{n}\right\| \rightarrow 0$ and $\left\|v_{n}-R_{n} v_{n}\right\| \rightarrow$ 0 . In fact, in view that $P_{S}$ and $I-\lambda_{n} M$ are nonexpansive, we can get that

$$
\begin{aligned}
\left\|v_{n+1}-v_{n}\right\|= & \left\|P_{S}\left(w_{n+1}-\lambda_{n+1} M w_{n+1}\right)-P_{S}\left(w_{n}-\lambda_{n} M w_{n}\right)\right\| \\
\leq & \left\|\left(w_{n+1}-\lambda_{n+1} M w_{n+1}\right)-\left(w_{n}-\lambda_{n} M w_{n}\right)\right\| \\
\leq & \left\|\left(w_{n+1}-\lambda_{n+1} M w_{n+1}\right)-\left(w_{n}-\lambda_{n+1} M w_{n}\right)\right\| \\
& +\left|\lambda_{n+1}-\lambda_{n}\right|\left\|M w_{n}\right\| \\
\leq & \left\|w_{n+1}-w_{n}\right\|+\left|\lambda_{n+1}-\lambda_{n}\right|\left\|M w_{n}\right\| .
\end{aligned}
$$

Hence,

$$
\begin{aligned}
\left\|w_{n+1}-w_{n}\right\|= & \| P_{S}\left[\alpha_{n} \gamma f\left(w_{n}\right)+\left(I-\alpha_{n} T\right) R_{n} v_{n}\right] \\
& -P_{S}\left[\alpha_{n-1} \gamma f\left(w_{n-1}\right)+\left(I-\alpha_{n-1} T\right) R_{n-1} v_{n-1}\right] \| \\
\leq & \|\left[\alpha_{n} \gamma f\left(w_{n}\right)+\left(I-\alpha_{n} T\right) R_{n} v_{n}\right] \\
& -\left[\alpha_{n-1} \gamma f\left(w_{n-1}\right)+\left(I-\alpha_{n-1} T\right) R_{n-1} v_{n-1}\right] \| \\
= & \| \alpha_{n} \gamma\left(f\left(w_{n}\right)-f\left(w_{n-1}\right)\right)+\gamma\left(\alpha_{n}-\alpha_{n-1}\right) \\
& \quad \times f\left(w_{n-1}\right)+\left(I-\alpha_{n} T\right)\left(R_{n} v_{n}-R_{n-1} v_{n-1}\right) \\
& +\left(\alpha_{n}-\alpha_{n-1}\right) T R_{n-1} v_{n-1} \| \\
\leq & \alpha_{n} \alpha \gamma\left\|w_{n}-w_{n-1}\right\|+\gamma\left|\alpha_{n}-\alpha_{n-1}\right|\left\|f\left(w_{n-1}\right)\right\| \\
& +\left(1-\alpha_{n} \bar{\gamma}\right)\left\|R_{n} v_{n}-R_{n-1} v_{n-1}\right\| \\
& +\left|\alpha_{n}-\alpha_{n-1}\right|\left\|T R_{n-1} v_{n-1}\right\|
\end{aligned}
$$




$$
\begin{aligned}
\leq & \alpha_{n} \alpha \gamma\left\|w_{n}-w_{n-1}\right\|+\gamma\left|\alpha_{n}-\alpha_{n-1}\right|\left\|f\left(w_{n-1}\right)\right\| \\
& +\left(1-\alpha_{n} \bar{\gamma}\right)\left\|R_{n} v_{n}-R_{n} v_{n-1}\right\| \\
& +\left(1-\alpha_{n} \bar{\gamma}\right)\left\|R_{n} v_{n-1}-R_{n-1} v_{n-1}\right\| \\
& +\left|\alpha_{n}-\alpha_{n-1}\right|\left\|T R_{n-1} v_{n-1}\right\| \\
\leq & \alpha_{n} \alpha \gamma\left\|w_{n}-w_{n-1}\right\|+\gamma\left|\alpha_{n}-\alpha_{n-1}\right|\left\|f\left(w_{n-1}\right)\right\| \\
& +\left(1-\alpha_{n} \bar{\gamma}\right)\left\|v_{n}-v_{n-1}\right\|+\left(1-\alpha_{n} \bar{\gamma}\right) \\
& \times\left\|R_{n} v_{n-1}-R_{n-1} v_{n-1}\right\| \\
& +\left|\alpha_{n}-\alpha_{n-1}\right|\left\|T R_{n-1} v_{n-1}\right\| \\
\leq & \alpha_{n} \alpha \gamma\left\|w_{n}-w_{n-1}\right\|+\gamma\left|\alpha_{n}-\alpha_{n-1}\right|\left\|f\left(w_{n-1}\right)\right\| \\
& +\left(1-\alpha_{n} \bar{\gamma}\right)\left\|w_{n}-w_{n-1}\right\| \\
& +\left(1-\alpha_{n} \bar{\gamma}\right)\left|\lambda_{n}-\lambda_{n-1}\right| \\
& \times\left\|M w_{n-1}\right\|+\left(1-\alpha_{n} \bar{\gamma}\right)\left\|R_{n} v_{n-1}-R_{n-1} v_{n-1}\right\| \\
& +\left|\alpha_{n}-\alpha_{n-1}\right|\left\|T R_{n-1} v_{n-1}\right\| \\
= & \left(1-\alpha_{n}(\bar{\gamma}-\alpha \gamma)\right)\left\|w_{n}-w_{n-1}\right\| \\
& +\gamma\left|\alpha_{n}-\alpha_{n-1}\right|\left\|f\left(w_{n-1}\right)\right\|+\left(1-\alpha_{n} \bar{\gamma}\right) \\
& \times\left|\lambda_{n}-\lambda_{n-1}\right|\left\|M w_{n-1}\right\|+\left(1-\alpha_{n} \bar{\gamma}\right) \\
& \times\left\|R_{n} v_{n-1}-R_{n-1} v_{n-1}\right\| \\
& +\left|\alpha_{n}-\alpha_{n-1}\right|\left\|T R_{n-1} v_{n-1}\right\| .
\end{aligned}
$$

Based on

$$
\begin{aligned}
& \left\|R_{n} v_{n-1}-R_{n-1} v_{n-1}\right\| \\
& \quad=\left\|P_{S}\left(I-\gamma_{n} G^{*} G\right) v_{n-1}-P_{S}\left(I-\gamma_{n-1} G^{*} G\right) v_{n-1}\right\| \\
& \quad \leq\left\|\left(I-\gamma_{n} G^{*} G\right) v_{n-1}-\left(I-\gamma_{n-1} G^{*} G\right) v_{n-1}\right\| \\
& \quad \leq\left|\gamma_{n}-\gamma_{n-1}\right|\|G\|^{2}\left\|v_{n-1}\right\|,
\end{aligned}
$$

we deduce that

$$
\begin{aligned}
\left\|w_{n+1}-w_{n}\right\| \leq & \left(1-\alpha_{n}(\bar{\gamma}-\alpha \gamma)\right)\left\|w_{n}-w_{n-1}\right\| \\
& +\gamma\left|\alpha_{n}-\alpha_{n-1}\right|\left\|f\left(w_{n-1}\right)\right\| \\
& +\left(1-\alpha_{n} \bar{\gamma}\right)\left|\lambda_{n}-\lambda_{n-1}\right|\left\|M w_{n-1}\right\| \\
& +\left(1-\alpha_{n} \bar{\gamma}\right)\left|\gamma_{n}-\gamma_{n-1}\right|\|G\|^{2}\left\|v_{n-1}\right\| \\
& +\left|\alpha_{n}-\alpha_{n-1}\right|\left\|T R_{n-1} v_{n-1}\right\| .
\end{aligned}
$$

By Lemma 3, we have

$$
\left\|w_{n+1}-w_{n}\right\| \longrightarrow 0
$$

On the other hand, since $u=P_{S}\left(u-\lambda_{n} M u\right)$ and $u=R_{n} u$ we have$$
\left\|w_{n+1}-u\right\|^{2}=\left\|P_{S}\left[\alpha_{n} \gamma f\left(w_{n}\right)+\left(I-\alpha_{n} T\right) R_{n} v_{n}\right]-P_{S}(u)\right\|^{2}
$$$$
\leq\left\|\alpha_{n} \gamma f\left(w_{n}\right)+\left(I-\alpha_{n} T\right) R_{n} v_{n}-u\right\|^{2}
$$$$
=\left\|\alpha_{n}\left(\gamma f\left(w_{n}\right)-T u\right)+\left(I-\alpha_{n} T\right)\left(R_{n} v_{n}-u\right)\right\|^{2}
$$$$
\leq\left(\left\|\alpha_{n}\left(\gamma f\left(w_{n}\right)-T u\right)\right\|\right.
$$$$
\left.+\left\|\left(I-\alpha_{n} T\right)\left(R_{n} v_{n}-u\right)\right\|\right)^{2}
$$$$
\leq \alpha_{n}\left\|\gamma f\left(w_{n}\right)-T u\right\|^{2}+\left\|\left(I-\alpha_{n} \bar{\gamma}\right) R_{n} v_{n}-u\right\|^{2}
$$$$
+2 \alpha_{n}\left(I-\alpha_{n} \bar{\gamma}\right)\left\|\gamma f\left(w_{n}\right)-T u\right\|\left\|R_{n} v_{n}-u\right\|
$$$$
\leq \alpha_{n}\left\|\gamma f\left(w_{n}\right)-T u\right\|^{2}+\left(I-\alpha_{n} \bar{\gamma}\right)\left\|v_{n}-u\right\|^{2}
$$$$
+2 \alpha_{n}\left(I-\alpha_{n} \bar{\gamma}\right)\left\|\gamma f\left(w_{n}\right)-T u\right\|\left\|v_{n}-u\right\|
$$$$
\leq \alpha_{n}\left\|\gamma f\left(w_{n}\right)-T u\right\|^{2}+\left(I-\alpha_{n} \bar{\gamma}\right)
$$$$
\times\left\|\left(I-\lambda_{n} M\right) w_{n}-\left(I-\lambda_{n} M\right) u\right\|^{2}
$$$$
+2 \alpha_{n}\left(I-\alpha_{n} \bar{\gamma}\right)\left\|\gamma f\left(w_{n}\right)-T u\right\|\left\|v_{n}-u\right\|
$$$$
\leq \alpha_{n}\left\|\gamma f\left(w_{n}\right)-T u\right\|^{2}+\left(I-\alpha_{n} \bar{\gamma}\right)
$$$$
\times\left(\left\|w_{n}-u\right\|^{2}-2 \lambda_{n}\left\langle w_{n}-u, M w_{n}-M u\right\rangle\right.
$$$$
\left.+\lambda_{n}^{2}\left\|M w_{n}-M u\right\|^{2}\right)+2 \alpha_{n}\left(I-\alpha_{n} \bar{\gamma}\right)
$$$$
\times\left\|\gamma f\left(w_{n}\right)-T u\right\|\left\|v_{n}-u\right\|
$$$$
\leq \alpha_{n}\left\|\gamma f\left(w_{n}\right)-T u\right\|^{2}
$$$$
+\left(I-\alpha_{n} \bar{\gamma}\right)\left(\left\|w_{n}-u\right\|^{2}-2 \lambda_{n} \beta\left\|M w_{n}-M u\right\|^{2}\right.
$$$$
\left.+\lambda_{n}^{2}\left\|M w_{n}-M u\right\|\right)
$$$$
+2 \alpha_{n}\left(I-\alpha_{n} \bar{\gamma}\right)\left\|\gamma f\left(w_{n}\right)-T u\right\|\left\|v_{n}-u\right\|
$$$$
\leq \alpha_{n}\left\|\gamma f\left(w_{n}\right)-T u\right\|^{2}+\left(I-\alpha_{n} \bar{\gamma}\right)
$$$$
\times\left(\left\|w_{n}-u\right\|^{2}+\lambda_{n}\left(\lambda_{n}-2 \beta\right)\left\|M w_{n}-M u\right\|^{2}\right)
$$$$
+2 \alpha_{n}\left(I-\alpha_{n} \bar{\gamma}\right)\left\|\gamma f\left(w_{n}\right)-T u\right\|\left\|v_{n}-u\right\|
$$$$
\leq \alpha_{n}\left\|\gamma f\left(w_{n}\right)-T u\right\|^{2}+\left\|w_{n}-u\right\|^{2}
$$$$
+\left(I-\alpha_{n} \bar{\gamma}\right) b(b-2 \beta)\left\|M w_{n}-M u\right\|^{2}
$$$$
+2 \alpha_{n}\left(I-\alpha_{n} \bar{\gamma}\right)\left\|\gamma f\left(w_{n}\right)-T u\right\|\left\|v_{n}-u\right\| .
$$

Therefore, we have

$$
\begin{aligned}
\left(I-\alpha_{n} \bar{\gamma}\right) b(2 \beta-b)\left\|M w_{n}-M u\right\|^{2} \\
\leq \alpha_{n}\left\|\gamma f\left(w_{n}\right)-T u\right\|^{2}+\left\|w_{n}-u\right\|^{2}-\left\|w_{n+1}-u\right\|^{2} \\
\quad+2 \alpha_{n}\left(I-\alpha_{n} \bar{\gamma}\right)\left\|\gamma f\left(w_{n}\right)-T u\right\|\left\|v_{n}-u\right\|
\end{aligned}
$$




$$
\begin{aligned}
= & \alpha_{n}\left\|\gamma f\left(w_{n}\right)-T u\right\|^{2}+2 \alpha_{n}\left(I-\alpha_{n} \bar{\gamma}\right) \\
& \times\left\|\gamma f\left(w_{n}\right)-T u\right\|\left\|v_{n}-u\right\|+\left(\left\|w_{n}-u\right\|-\left\|w_{n+1}-u\right\|\right) \\
& \times\left(\left\|w_{n}-u\right\|-\left\|w_{n+1}-u\right\|\right) \\
\leq & \alpha_{n}\left\|\gamma f\left(w_{n}\right)-T u\right\|^{2} \\
& +2 \alpha_{n}\left(I-\alpha_{n} \bar{\gamma}\right)\left\|\gamma f\left(w_{n}\right)-T u\right\|\left\|v_{n}-u\right\| \\
& +\left\|w_{n}-w_{n+1}\right\|\left(\left\|w_{n}-u\right\|-\left\|w_{n+1}-u\right\|\right) .
\end{aligned}
$$

Since $\alpha_{n} \rightarrow 0,\left\|w_{n}-w_{n+1}\right\| \rightarrow 0$, we can get that

$$
\left\|M w_{n}-M u\right\| \longrightarrow 0
$$

Furthermore, by the property of the projection $P_{S}$, we have

$$
\begin{aligned}
\left\|v_{n}-u\right\|= & \left\|P_{S}\left(I-\lambda_{n} M\right) w_{n}-P_{S}\left(I-\lambda_{n} M\right) u\right\| \\
\leq & \left\langle\left(w_{n}-\lambda_{n} M w_{n}\right)-\left(u-\lambda_{n} M u\right), v_{n}-u\right\rangle \\
= & \frac{1}{2}\left(\left\|\left(w_{n}-\lambda_{n} M w_{n}\right)-\left(u-\lambda_{n} M u\right)\right\|^{2}+\left\|v_{n}-u\right\|^{2}\right. \\
& \left.\quad-\left\|\left(w_{n}-\lambda_{n} M w_{n}\right)-\left(u-\lambda_{n} M u\right)-\left(v_{n}-u\right)\right\|^{2}\right) \\
\leq & \frac{1}{2}\left(\left\|w_{n}-u\right\|^{2}+\left\|v_{n}-u\right\|^{2}\right. \\
& \left.\quad-\left\|\left(w_{n}-v_{n}\right)-\lambda_{n}\left(M w_{n}-M u\right)\right\|^{2}\right) \\
\leq & \left\|w_{n}-u\right\|^{2}-\left\|w_{n}-v_{n}\right\|^{2} \\
& +2 \lambda_{n}\left\langle w_{n}-v_{n}, M w_{n}-M u\right\rangle-\lambda_{n}^{2}\left\|M w_{n}-M u\right\|^{2} .
\end{aligned}
$$

Then, we have

$$
\begin{aligned}
\left\|w_{n+1}-u\right\|^{2}= & \left\|P_{S}\left[\alpha_{n} \gamma f\left(w_{n}\right)+\left(I-\alpha_{n} T\right) R_{n} v_{n}\right]-P_{S}(u)\right\|^{2} \\
\leq & \alpha_{n}\left\|\gamma f\left(w_{n}\right)-T u\right\|^{2}+\left(1-\alpha_{n} \bar{\gamma}\right)\left\|v_{n}-u\right\|^{2} \\
& +2 \alpha_{n}\left(I-\alpha_{n} \bar{\gamma}\right)\left\|\gamma f\left(w_{n}\right)-T u\right\|\left\|v_{n}-u\right\| \\
\leq & \alpha_{n}\left\|\gamma f\left(w_{n}\right)-T u\right\|^{2}+\left(1-\alpha_{n} \bar{\gamma}\right)\left\|w_{n}-u\right\|^{2} \\
& -\left(1-\alpha_{n} \bar{\gamma}\right)\left\|w_{n}-v_{n}\right\|^{2}+2\left(1-\alpha_{n} \bar{\gamma}\right) \\
& \times \lambda_{n}\left\langle w_{n}-v_{n}, M w_{n}-M u\right\rangle-\left(1-\alpha_{n} \bar{\gamma}\right) \\
& \times \lambda_{n}^{2}\left\|M w_{n}-M u\right\|^{2}+2 \alpha_{n}\left(I-\alpha_{n} \bar{\gamma}\right) \\
& \times\left\|\gamma f\left(w_{n}\right)-T u\right\|\left\|v_{n}-u\right\|,
\end{aligned}
$$

which yields that

$$
\begin{aligned}
\left(1-\alpha_{n} \bar{\gamma}\right) & \left\|w_{n}-v_{n}\right\|^{2} \\
\leq & \alpha_{n}\left\|\gamma f\left(w_{n}\right)-T u\right\|^{2} \\
& +\left(\left\|w_{n}-u\right\|+\left\|w_{n+1}-u\right\|\right)\left\|w_{n}-w_{n+1}\right\| \\
& +2\left(1-\alpha_{n} \bar{\gamma}\right) \lambda_{n}\left\langle w_{n}-v_{n}, M w_{n}-M u\right\rangle \\
& -\left(1-\alpha_{n} \bar{\gamma}\right) \lambda_{n}^{2}\left\|M w_{n}-M u\right\|^{2} \\
& +2 \alpha_{n}\left(I-\alpha_{n} \bar{\gamma}\right)\left\|\gamma f\left(w_{n}\right)-T u\right\|\left\|v_{n}-u\right\| .
\end{aligned}
$$

Since $\alpha_{n} \rightarrow 0,\left\|w_{n}-w_{n+1}\right\| \rightarrow 0$, and $\left\|M w_{n}-M u\right\| \rightarrow 0$, we can get that

$$
\left\|w_{n}-v_{n}\right\| \longrightarrow 0 .
$$

Next, since $\alpha_{n} \rightarrow 0$ we can get that

$$
\begin{aligned}
\| w_{n+1} & -R_{n} v_{n} \| \\
& =\left\|P_{S}\left[\alpha_{n} \gamma f\left(w_{n}\right)+\left(I-\alpha_{n} T\right) R_{n} v_{n}\right]-P_{S}\left(R_{n} v_{n}\right)\right\| \\
& \leq\left\|\alpha_{n} \gamma f\left(w_{n}\right)+\left(I-\alpha_{n} T\right) R_{n} v_{n}-R_{n} v_{n}\right\| \\
& =\alpha_{n}\left\|\gamma f\left(w_{n}\right)-T R_{n} v_{n}\right\| \longrightarrow 0 .
\end{aligned}
$$

Hence,

$$
\begin{aligned}
\left\|w_{n}-R_{n} v_{n}\right\| \leq & \left\|w_{n}-w_{n+1}\right\|+\left\|w_{n+1}-R_{n} v_{n}\right\| \longrightarrow 0, \\
\left\|w_{n}-R_{n} w_{n}\right\| & \leq\left\|w_{n}-R_{n} v_{n}\right\|+\left\|R_{n} v_{n}-R_{n} w_{n}\right\| \\
& \leq\left\|w_{n}-R_{n} v_{n}\right\|+\left\|v_{n}-w_{n}\right\| \longrightarrow 0, \\
\left\|v_{n}-R_{n} v_{n}\right\| & \leq\left\|v_{n}-w_{n}\right\|+\left\|w_{n}-R_{n} v_{n}\right\| \longrightarrow 0 .
\end{aligned}
$$

Let the mapping $R$ be defined by

$$
R w=\lim _{n \rightarrow \infty} R_{n} w, \quad \forall w \in H .
$$

By Lemma 5 we can get that $\operatorname{Fix}(R)=\cap \operatorname{Fix}\left(R_{n}\right)$ and

$$
\begin{aligned}
\left\|R w_{n}-w_{n}\right\| \leq & \left\|R w_{n}-R v_{n}\right\|+\left\|R v_{n}-R_{n} v_{n}\right\| \\
& +\left\|R_{n} v_{n}-w_{n}\right\| \\
\leq & \left\|w_{n}-v_{n}\right\| \\
& +\sup \left\{\left\|R_{n} z-R z\right\|, z \in\left\{v_{n}\right\}\right\} \\
& +\left\|R_{n} v_{n}-w_{n}\right\| \\
& \longrightarrow
\end{aligned}
$$

Note that it is easy to check that $P_{\operatorname{InVI}(S, M)}(\gamma f+I-T)$ is a contraction; by Banach contraction mapping principle, $P_{\Gamma \cap V I(S, M)}(\gamma f+I-T)$ has a unique fixed point $p \in H$. By the property of the projection $P_{\mathrm{\Gamma} \cap \mathrm{VI}(S, M)}$, we can get that

$$
\langle(\gamma f-T) p, w-p\rangle \leq 0, \quad \forall w \in \Gamma \cap \operatorname{VI}(S, M) .
$$


Choose a subsequence $v_{n k}$ of $v_{n}$ such that

$$
\begin{aligned}
\limsup _{n \rightarrow \infty} & \left\langle(\gamma f-T) p, R_{n} v_{n}-p\right\rangle \\
& =\lim _{n \rightarrow \infty}\left\langle(\gamma f-T) p, R_{n k} v_{n k}-p\right\rangle .
\end{aligned}
$$

Since $\left\{v_{n}\right\}$ is bounded, there exists a subsequence $\left\{v_{n k_{i}}\right\}$ of $\left\{v_{n k}\right\}$ which converges weakly $q$. Without loss of generality, we may assume that $v_{n k}$ converges weakly $q$. Note that, by $\left\|v_{n}-R_{n} v_{n}\right\| \rightarrow 0$, we can get $R_{n k} v_{n k}$ converges weakly $q$. Furthermore, $\left\|w_{n}-R w_{n}\right\| \rightarrow 0$, $\left\|v_{n}-w_{n}\right\| \rightarrow 0$; by Lemmas 2 and 5, we obtain that $q \in \operatorname{Fix}(R)=\cap \operatorname{Fix}\left(R_{n}\right)$.

Next we will prove that $q \in \operatorname{VI}(S, M)$.

Choose $v \in S$, and let $w_{v} \in\{w \in H,\langle v-u, w\rangle \geq 0, \forall u \in$ $S\}$. Since $v_{n} \in S$, we can get $\left\langle v-v_{n}, w_{v}\right\rangle \geq 0$. On the other hand, by the property of the projection $P_{S}$ and $v_{n}=P_{S}\left(w_{n}-\lambda_{n} M w_{n}\right)$, we have

$$
\begin{gathered}
\left\langle v-v_{n}, v_{n}-\left(w_{n}-\lambda_{n} M w_{n}\right)\right\rangle \geq 0, \\
\left\langle v-v_{n}, \frac{v_{n}-w_{n}}{\lambda_{n}}+M w_{n}\right\rangle \geq 0 .
\end{gathered}
$$

It follows that

$$
\begin{aligned}
\left\langle v-v_{n k}, M v+w_{v}\right\rangle \geq & \left\langle v-v_{n k}, M v\right\rangle \\
\geq & \left\langle v-v_{n k}, M v\right\rangle \\
& -\left\langle v-v_{n k}, \frac{v_{n k}-w_{n k}}{\lambda_{n k}}+M w_{n k}\right\rangle \\
= & \left\langle v-v_{n k}, M v-M w_{n k}-\frac{v_{n k}-w_{n k}}{\lambda_{n k}}\right\rangle \\
= & \left\langle v-v_{n k}, M v-M v_{n k}\right\rangle \\
& +\left\langle v-v_{n k}, M v_{n k}-M w_{n k}\right\rangle \\
& -\left\langle v-v_{n k}, \frac{v_{n k}-w_{n k}}{\lambda_{n k}}\right\rangle \\
\geq & \left\langle v-v_{n k}, M v_{n k}-M w_{n k}\right\rangle \\
& -\left\langle v-v_{n k}, \frac{v_{n k}-w_{n k}}{\lambda_{n k}}\right\rangle .
\end{aligned}
$$

Since $\left\|M v_{n}-M w_{n}\right\| \leq\left\|v_{n}-w_{n}\right\| \rightarrow 0$ and $v_{n k}$ converges weakly $q$, we can get that $\left\langle v-q, M v+w_{v}\right\rangle \geq 0$.

Note that $M$ is a $\beta$-inverse strongly monotone mapping, and if $w_{v} \in\{w \in H,\langle v-u, w\rangle \geq 0, \forall u \in S\}$, then $\alpha w_{v} \in$ $\{w \in H,\langle v-u, w\rangle \geq 0, \forall u \in S\}$ for all $\alpha>0$. Hence, we can choose $w_{v} \in\{w \in H,\langle v-u, w\rangle \geq 0, \forall u \in S\}$ such that $\left\langle v-q, w_{v}\right\rangle \geq\langle v-q, M v-M q\rangle$. By

$$
\left\langle v-q, M v+w_{v}\right\rangle \geq 0,
$$

we can get that

$$
\langle v-q, M v-M q\rangle+\left\langle v-q, M q-w_{v}\right\rangle \geq 0 .
$$

Hence

$$
\langle v-q, M q\rangle \geq\left\langle v-q, w_{v}\right\rangle-\langle v-q, M v-M q\rangle \geq 0 .
$$

It follows that $q \in \mathrm{VI}(S, M)$. We obtain that $q \in P_{\Gamma \cap \mathrm{VI}(S, M)}$ and $\langle(\gamma f-T) p, q-p\rangle \leq 0$, for all $w \in \Gamma \cap \operatorname{VI}(S, M)$.

Finally, we prove that $\lim _{n \rightarrow \infty}\left\|w_{n}-q\right\|=0$. Since $\| v_{n}-$ $u\|\leq\| w_{n}-u \|$, we have

$$
\begin{aligned}
& \left\|w_{n+1}-p\right\|^{2}=\left\|P_{S}\left[\alpha_{n} \gamma f\left(w_{n}\right)+\left(I-\alpha_{n} T\right) R_{n} v_{n}\right]-P_{S}(p)\right\|^{2} \\
& \leq\left\|\alpha_{n}\left(\gamma f\left(w_{n}\right)-T p\right)+\left(I-\alpha_{n} T\right)\left(R_{n} v_{n}-p\right)\right\|^{2} \\
& \leq\left\|\left(I-\alpha_{n} T\right)\left(R_{n} v_{n}-p\right)\right\|^{2}+\alpha_{n}^{2}\left\|\gamma f\left(w_{n}\right)-T q\right\|^{2} \\
& +2 \alpha_{n}\left\langle\left(I-\alpha_{n} T\right)\left(R_{n} v_{n}-p\right), \gamma f\left(w_{n}\right)-T q\right\rangle \\
& \leq\left(1-\alpha_{n} \bar{\gamma}\right)^{2}\left\|v_{n}-p\right\|^{2}+\alpha_{n}^{2}\left\|\gamma f\left(w_{n}\right)-T q\right\|^{2} \\
& +2 \alpha_{n}\left\langle R_{n} v_{n}-p, \gamma f\left(w_{n}\right)-T p\right\rangle \\
& -2 \alpha_{n}^{2}\left\langle T\left(R_{n} v_{n}-p\right), \gamma f\left(w_{n}\right)-T p\right\rangle \\
& \leq\left(1-\alpha_{n} \bar{\gamma}\right)^{2}\left\|v_{n}-p\right\|^{2}+\alpha_{n}^{2}\left\|\gamma f\left(w_{n}\right)-T q\right\|^{2} \\
& +2 \alpha_{n}\left\langle R_{n} v_{n}-p, \gamma f\left(w_{n}\right)-\gamma f(p)\right\rangle \\
& +2 \alpha_{n}\left\langle R_{n} v_{n}-p, \gamma f(p)-T p\right\rangle \\
& -2 \alpha_{n}^{2}\left\langle T\left(R_{n} v_{n}-p\right), \gamma f\left(w_{n}\right)-T p\right\rangle \\
& \leq\left(1-\alpha_{n} \bar{\gamma}\right)^{2}\left\|v_{n}-p\right\|^{2}+\alpha_{n}^{2}\left\|\gamma f\left(w_{n}\right)-T q\right\|^{2} \\
& +2 \alpha_{n}\left\|R_{n} v_{n}-p\right\|\left\|\gamma f\left(w_{n}\right)-\gamma f(p)\right\| \\
& +2 \alpha_{n}\left\langle R_{n} v_{n}-p, \gamma f(p)-T p\right\rangle \\
& -2 \alpha_{n}^{2}\left\langle T\left(R_{n} v_{n}-p\right), \gamma f\left(w_{n}\right)-T p\right\rangle \\
& \leq\left(1-\alpha_{n} \bar{\gamma}\right)^{2}\left\|v_{n}-p\right\|^{2}+\alpha_{n}^{2}\left\|\gamma f\left(w_{n}\right)-T q\right\|^{2} \\
& +2 \gamma \alpha \alpha_{n}\left\|v_{n}-p\right\|\left\|w_{n}-p\right\| \\
& +2 \alpha_{n}\left\langle R_{n} v_{n}-p, \gamma f(p)-T p\right\rangle \\
& -2 \alpha_{n}^{2}\left\langle T\left(R_{n} v_{n}-p\right), \gamma f\left(w_{n}\right)-T p\right\rangle \\
& \leq\left(1-\alpha_{n} \bar{\gamma}\right)^{2}\left\|v_{n}-p\right\|^{2}+\alpha_{n}^{2}\left\|\gamma f\left(w_{n}\right)-T q\right\|^{2} \\
& +2 \gamma \alpha \alpha_{n}\left\|w_{n}-p\right\|^{2} \\
& +2 \alpha_{n}\left\langle R_{n} v_{n}-p, \gamma f(p)-T p\right\rangle \\
& -2 \alpha_{n}^{2}\left\langle T\left(R_{n} v_{n}-p\right), \gamma f\left(w_{n}\right)-T p\right\rangle \\
& \leq\left[\left(1-\alpha_{n} \bar{\gamma}\right)^{2}+2 \gamma \alpha \alpha_{n}\right]\left\|w_{n}-p\right\|^{2} \\
& +\alpha_{n}\left[2\left\langle R_{n} v_{n}-p, \gamma f(p)-T p\right\rangle\right. \\
& +\alpha_{n}\left\|\gamma f\left(w_{n}\right)-T q\right\|^{2} \\
& \left.+2 \alpha_{n}\left\|T\left(R_{n} v_{n}-p\right)\right\|\left\|\gamma f\left(w_{n}\right)-T p\right\|\right]
\end{aligned}
$$




$$
\begin{aligned}
=[1- & \left.2(\bar{\gamma}-\gamma \alpha) \alpha_{n}\right]\left\|w_{n}-p\right\|^{2} \\
+\alpha_{n} & {\left[2\left\langle R_{n} v_{n}-p, \gamma f(p)-T p\right\rangle\right.} \\
& +\alpha_{n}\left\|\gamma f\left(w_{n}\right)-T q\right\|^{2}+2 \alpha_{n}\left\|T\left(R_{n} v_{n}-p\right)\right\| \\
& \left.\times\left\|\gamma f\left(w_{n}\right)-T p\right\|+\alpha_{n} \bar{\gamma}^{2}\left\|w_{n}-p\right\|^{2}\right] .
\end{aligned}
$$

Since $\left\{w_{n}\right\},\left\{f\left(w_{n}\right)\right\}, \quad\left\{R_{n} v_{n}\right\}$, and $\limsup _{n \rightarrow \infty}\langle(\gamma f-$ $\left.T) p, R_{n} v_{n}-p\right\rangle \leq 0$, using Lemma 3 , we can conclude that

$$
\lim _{n \rightarrow \infty}\left\|w_{n}-q\right\|=0
$$

which completes the proof.

By Lemma 1 , we know that $G^{*} G$ is $1 /\|G\|^{2}$-inverse strongly monotone. Hence, letting $M=G^{*} G$, we can get the following Corollary.

Corollary 7. Let $\Gamma$ denote the solutions set of the SEP, $S=C \times$ $Q, f$ a contraction on $H=H_{1} \times H_{2}$ with coefficient $0<\alpha<1, a$ strongly positive linear bounded operator $T$ with coefficient $\bar{\gamma}>$ 0 . Assume that $0<\gamma<\bar{\gamma} / \alpha, \Gamma \neq \emptyset$, and $\left\{w_{n}\right\}=\left\{\left(x_{n}, y_{n}\right)\right\} \subseteq H$ is the sequence generated by the following algorithm: $w_{0} \in H$,

$$
\begin{aligned}
w_{n+1}=P_{S}[ & \alpha_{n} \gamma f\left(w_{n}\right)+\left(I-\alpha_{n} T\right) P_{S} \\
& \left.\times\left(I-\gamma_{n} G^{*} G\right) P_{S}\left(w_{n}-\lambda_{n} G^{*} G w_{n}\right)\right]
\end{aligned}
$$

for all $n=0,1,2, \ldots$, where $\alpha_{n} \in(0,1), \lambda_{n}, \gamma_{n} \in\left(0,2 /\|G\|^{2}\right)$. If $\alpha_{n}, \lambda_{n}$, and $\gamma_{n}$ are chosen so that $\lambda_{n} \in[a, b]$ for some $a, b$ with $0<a<b<2 /\|G\|^{2}$ and

(i) $\lim _{n} \alpha_{n}=0$,

(ii) $\sum_{n=0}^{\infty} \alpha_{n}=\infty$,

(iii) $\sum_{n=0}^{\infty}\left|\alpha_{n+1}-\alpha_{n}\right|<\infty$,

(v) $\sum_{n=0}^{\infty}\left|\lambda_{n+1}-\lambda_{n}\right|<\infty$,

(iv) $\sum_{n=0}^{\infty}\left|\gamma_{n+1}-\gamma_{n}\right|<\infty$,

then the sequence $\left\{w_{n}\right\}$ converges strongly to $\widetilde{w}$ which solves the SEP and the following variational inequality: $\langle(T-\lambda f) \widetilde{w}, w-$ $\widetilde{w}\rangle \geq 0$ for all $w \in S$.

Furthermore, if $M=G^{*} G$ and $\gamma_{n}=0$, changed slightly for Theorem 6, we get the following result.

Corollary 8. Let $\Gamma$ denote the solutions set of the SEP, $S=C \times$ $Q, f$ a contraction on $H=H_{1} \times H_{2}$ with coefficient $0<\alpha<1, a$ strongly positive linear bounded operator $T$ with coefficient $\bar{\gamma}>$ 0 . Assume that $0<\gamma<\bar{\gamma} / \alpha, \Gamma \neq \emptyset$, and $\left\{w_{n}\right\}=\left\{\left(x_{n}, y_{n}\right)\right\} \subseteq H$ is the sequence generated by the following algorithm: $w_{0} \in H$,

$$
w_{n+1}=\alpha_{n} \gamma f\left(w_{n}\right)+\left(I-\alpha_{n} T\right) P_{S}\left(w_{n}-\lambda_{n} G^{*} G w_{n}\right)
$$

for all $n=0,1,2, \ldots$, where $\alpha_{n} \in(0,1), \lambda_{n} \in\left(0,2 /\|G\|^{2}\right)$. If $\alpha_{n}$, $\lambda_{n}$ are chosen so that $\lambda_{n} \in[a, b]$ for some $a, b$ with $0<a<b<$ $2 /\|G\|^{2}$ and

(i) $\lim _{n} \alpha_{n}=0$,

(ii) $\sum_{n=0}^{\infty} \alpha_{n}=\infty$,

(iii) $\sum_{n=0}^{\infty}\left|\alpha_{n+1}-\alpha_{n}\right|<\infty$,

(v) $\sum_{n=0}^{\infty}\left|\lambda_{n+1}-\lambda_{n}\right|<\infty$,

then the sequence $\left\{w_{n}\right\}$ converges strongly to $\widetilde{w}$ which solves the SEP and the following variational inequality: $\langle(T-\lambda f) \widetilde{w}, w-$ $\widetilde{w}\rangle \geq 0$ for all $w \in S$.

\section{Acknowledgments}

This research was supported by NSFC Grants nos. 11071279, 11226125 , and 11301379 .

\section{References}

[1] Y. Censor and T. Elfving, "A multiprojection algorithm using Bregman projections in a product space," Numerical Algorithms, vol. 8, no. 2-4, pp. 221-239, 1994.

[2] C. Byrne, "Iterative oblique projection onto convex sets and the split feasibility problem," Inverse Problems, vol. 18, no. 2, pp. 441453, 2002.

[3] C. Byrne, "A unified treatment of some iterative algorithms in signal processing and image reconstruction," Inverse Problems, vol. 20, no. 1, pp. 103-120, 2004.

[4] B. Qu and N. Xiu, "A note on the CQ algorithm for the split feasibility problem," Inverse Problems, vol. 21, no. 5, pp. 16551665,2005

[5] H.-K. Xu, "A variable Krasnosel'skii-Mann algorithm and the multiple-set split feasibility problem," Inverse Problems, vol. 22, no. 6, pp. 2021-2034, 2006.

[6] Q. Yang, "The relaxed CQ algorithm solving the split feasibility problem," Inverse Problems, vol. 20, no. 4, pp. 1261-1266, 2004.

[7] Q. Yang and J. Zhao, "Generalized KM theorems and their applications," Inverse Problems, vol. 22, no. 3, pp. 833-844, 2006.

[8] A. Moudafi, "A relaxed alternating CQ-algorithm for convex feasibility problems," Nonlinear Analysis: Theory, Methods \& Applications, vol. 79, pp. 117-121, 2013.

[9] L. Y. Shi, R. D. Chen, and Y. J. Wu, "Strong convergence of iterative algorithms for the split equality problem," Inverse Problems. In press.

[10] G. Ronald, Banach Algebra Techniques in Operator Theory, Springer, New York, NY, USA, 1972.

[11] K. Goebel and W. A. Kirk, Topics in Metric Fixed Point Theory, vol. 28 of Cambridge Studies in Advanced Mathematics, Cambridge University Press, Cambridge, Mass, USA, 1990.

[12] K. Geobel and S. Reich, Uniform Convexity, Nonexpansive Mappings, and Hyperbolic Geometry, Dekker, New York, NY, USA, 1984.

[13] K. Aoyama, Y. Kimura, W. Takahashi, and M. Toyoda, "Approximation of common fixed points of a countable family of nonexpansive mappings in a Banach space," Nonlinear Analysis: Theory, Methods \& Applications, vol. 67, no. 8, pp. 2350-2360, 2007.

[14] G. Marino and H.-K. Xu, "A general iterative method for nonexpansive mappings in Hilbert spaces," Journal of Mathematical Analysis and Applications, vol. 318, no. 1, pp. 43-52, 2006. 


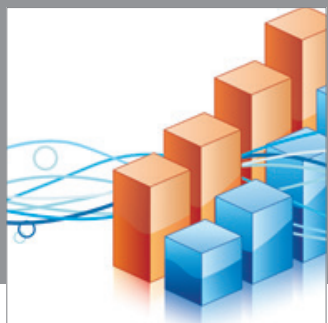

Advances in

Operations Research

mansans

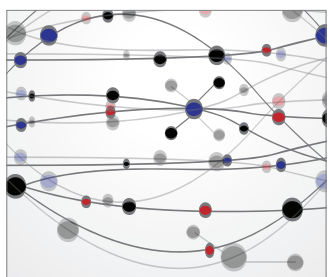

The Scientific World Journal
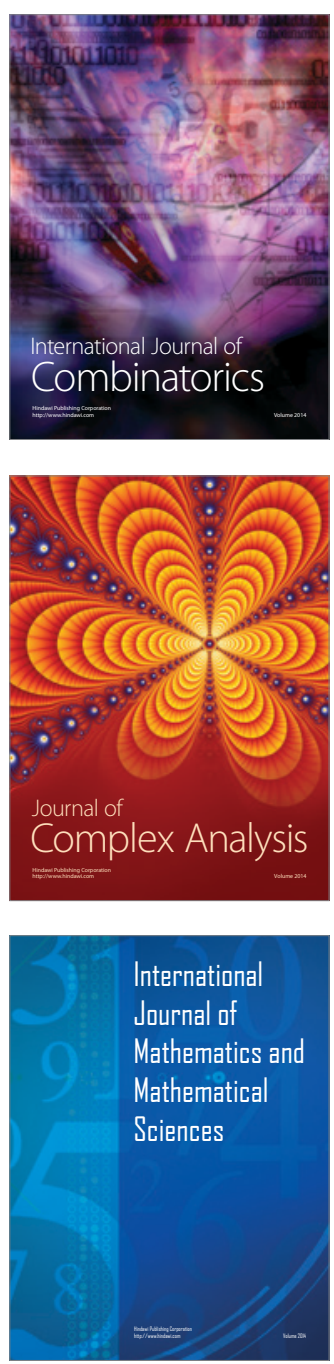
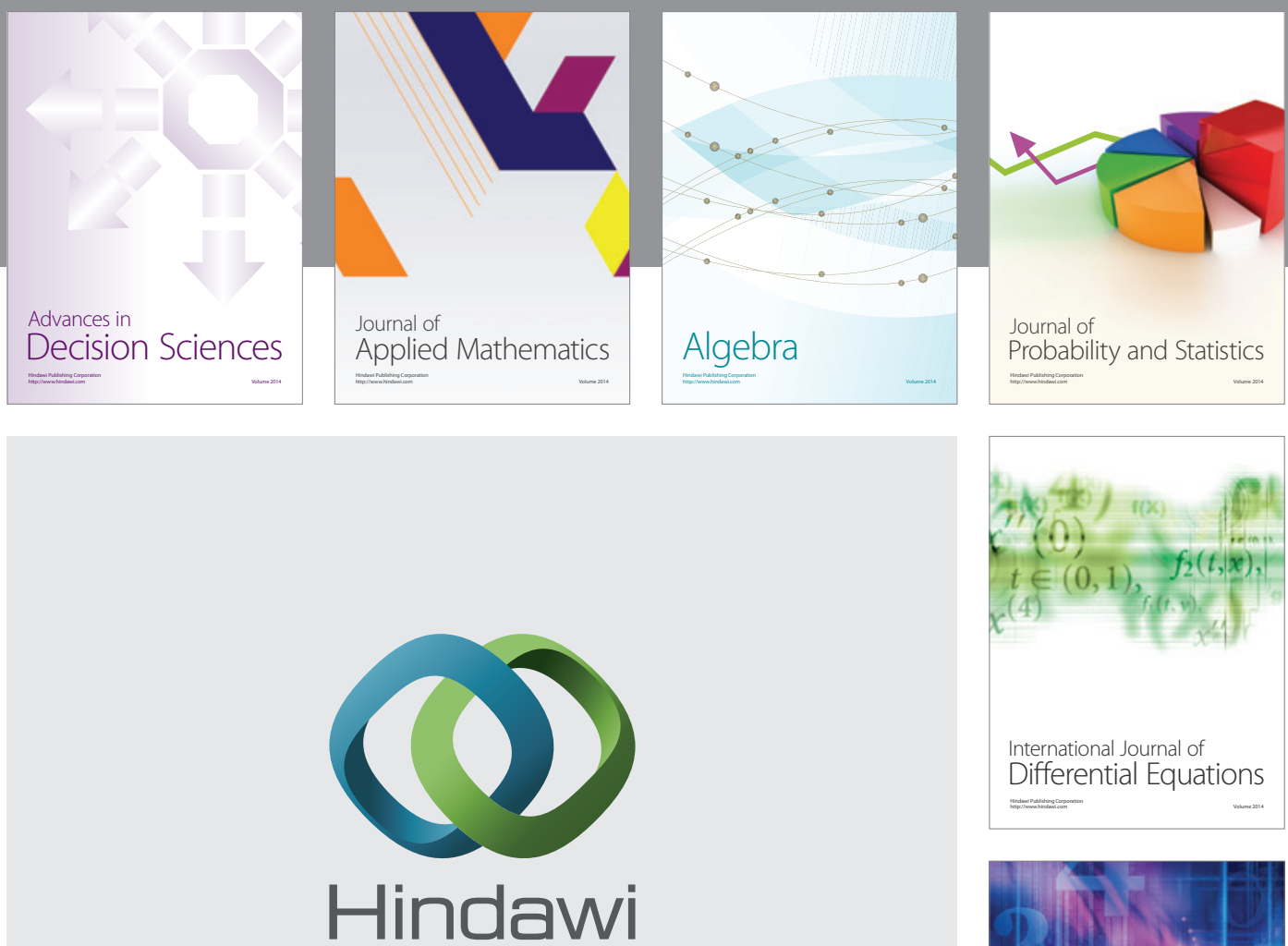

Submit your manuscripts at http://www.hindawi.com
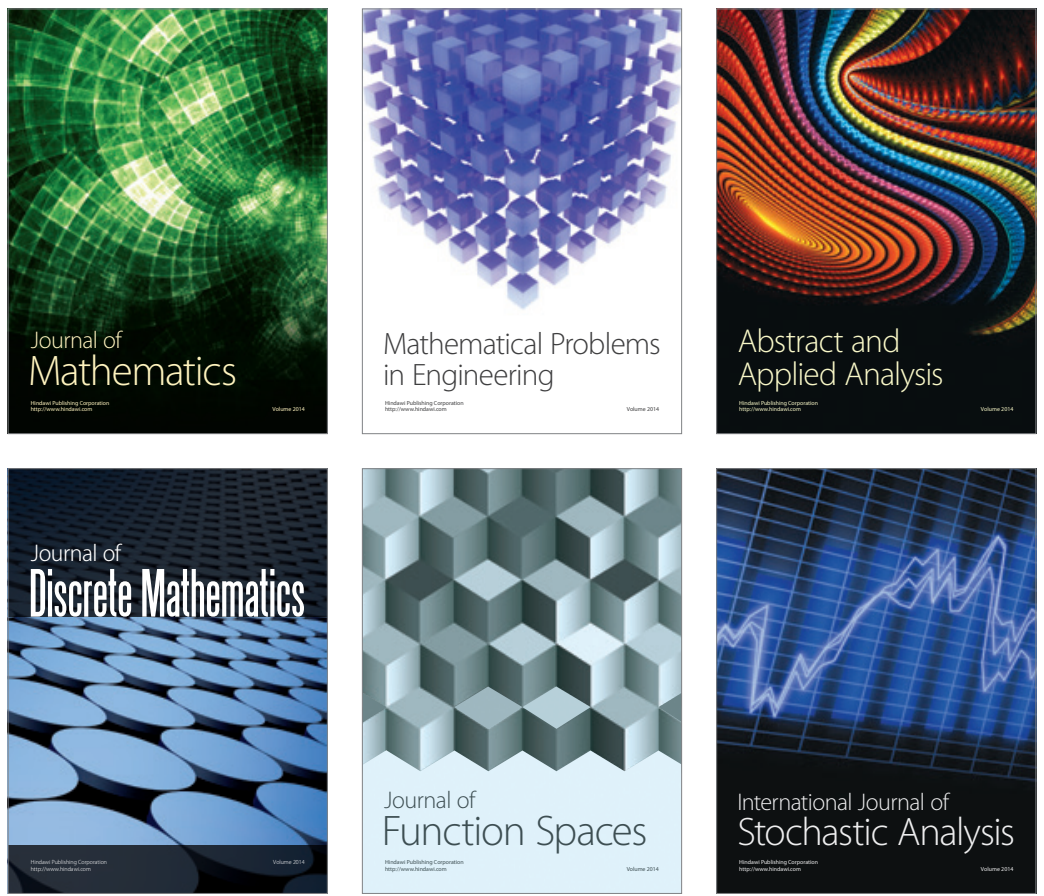

Journal of

Function Spaces

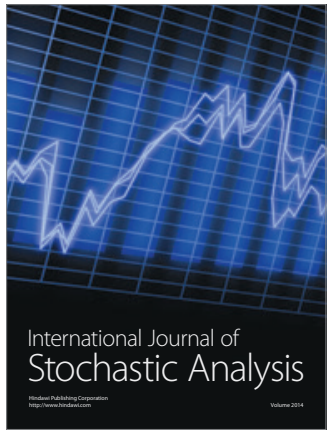

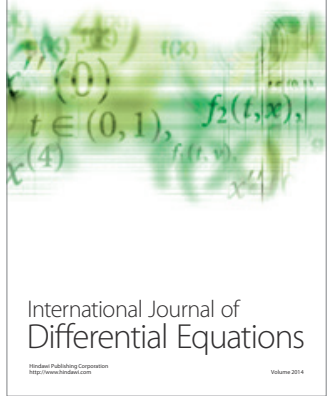
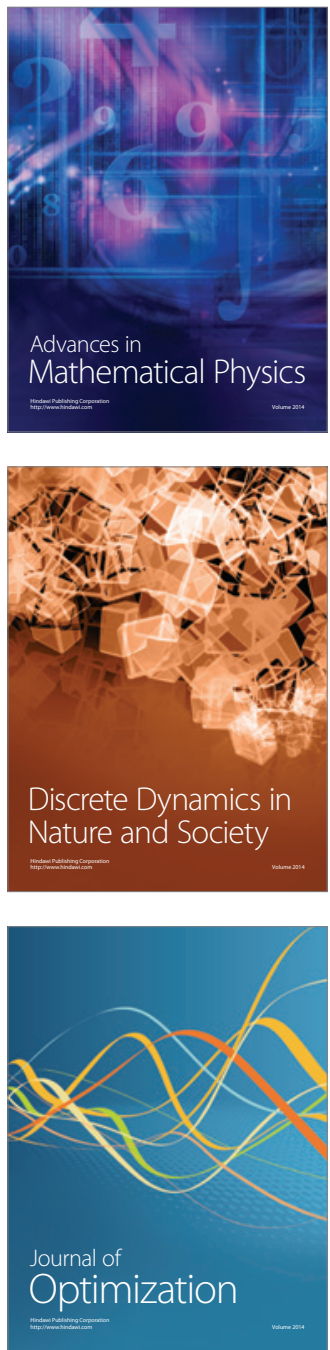